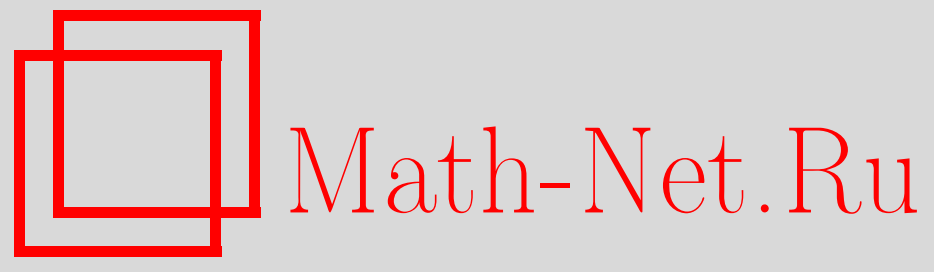

О. Ф. Меньших, О законах сохранения второго порядка для уравнения Борна-Инфельда и других родственных уравнений, Матем. заметки, 2008, том 84, выпуск $6,874-881$

DOI: https://doi.org/10.4213/mzm4443

Использование Общероссийского математического портала Math-Net.Ru подразумевает, что вы прочитали и согласны с пользовательским соглашением http://www.mathnet.ru/rus/agreement

Параметры загрузки:

IP : 18.207 .199 .55

26 апреля 2023 г., 13:00:43

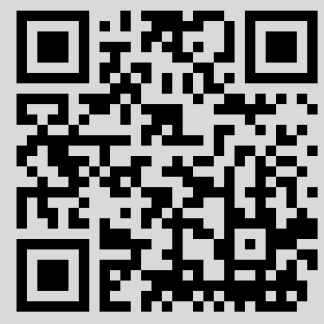


Том 84 выпуск 6 декабрь 2008

УДК 517.95

\section{О законах сохранения второго порядка для уравнения Борна-Инфельда и других родственных уравнений}

\section{О. Ф. Меньших}

Описан класс квазилинейных уравнений с частными производными второго порядка с двумя независимыми переменными в общем случае смешанного типа, для которого построены законы сохранения второго порядка, квадратичные относительно вторых производных. В качестве примеров приведены подобные законы сохранения для уравнения Борна-Инфельда, для уравнения минимальных и максимальных поверхностей в пространстве Минковского и для классического уравнения минимальных поверхностей.

Библиография: 17 названий.

Введение. Данная работа является продолжением статьи [1], в которой рассматривались законы сохранения [2], [3] первого порядка вида

$$
\frac{\partial \varphi\left(U_{t}, U_{x}\right)}{\partial t}+\frac{\partial \psi\left(U_{t}, U_{x}\right)}{\partial x}=0
$$

для уравнения Борна-Инфельда

$$
L(U)=\left(1+U_{x}^{2}\right) U_{t t}-2 U_{x} \cdot U_{t} \cdot U_{x t}+\left(U_{t}^{2}-1\right) U_{x x}=0
$$

и родственных уравнений вида

$$
U_{t t}=2 A_{13}(p, \lambda) U_{t x}+A_{11}(p, \lambda) U_{x x}, \quad p=U_{x}, \quad \lambda=U_{t} .
$$

Целью данной статьи является построение законов сохранения второго порядка

$$
\frac{\partial}{\partial t}\left[E\left(p, \lambda, U_{x x}, U_{x t}\right)\right]+\frac{\partial\left[G(p, \lambda), U_{x x}, U_{x t}\right]}{\partial x}=0
$$

для уравнения (2) и более широкого класса родственных уравнений (3). Будем предполагать, что функции $E$ и $G$ являются квадратичными формами по $U_{x x}, U_{x t}$.

Будем рассматривать класс квазилинейных уравнений (3) в общем случае смешанного типа. Предполагаем, что коэффициенты уравнения (3) являются достаточно гладкими функциями переменных $p, \lambda$ в некоторой области $D(p, \lambda)$, в которой

$$
\frac{\partial\left(A_{11}, A_{13}\right)}{\partial(p, \lambda)} \neq 0 .
$$

Введем необходимые для дальнейшего изложения определения. 
ОПРедЕЛЕниЕ 1 [1], [4]. Квазилинейные уравнения с частными производными (3), решения которых рассматриваются в гиперболической области

$$
A_{13}^{2}+A_{11}>0
$$

а коэффициенты удовлетворяют условиям (5) и дополнительным условиям

$$
\begin{aligned}
\frac{\partial A_{11}}{\partial p} & =-2 A_{11} \frac{\partial A_{13}}{\partial \lambda}, \\
\frac{\partial A_{11}}{\partial \lambda} & =-\left(2 \frac{\partial A_{13}}{\partial p}+4 A_{13} \frac{\partial A_{13}}{\partial \lambda}\right),
\end{aligned}
$$

будем называть $A$-гиперболическими уравнениями.

ОПРЕДЕЛЕниЕ 2 [5]. Квазилинейные уравнения с частными производными (3), решения которых рассматриваются в эллиптической области

$$
A_{13}^{2}+A_{11}<0,
$$

а коэффициенты удовлетворяют условию (5) и тем же условиям (7), будем называть A-эллиптическими уравнениями.

Введенные классы уравнений приводятся к специальным каноническим системам [5], [6]. Здесь только отметим, что любое $A$-гиперболическое уравнение приводится к слаболинейной [7] системе квазилинейных уравнений первого порядка, записанной в инвариантах Римана [1], [4]

$$
\frac{\partial r_{1}}{\partial t}+r_{2} \frac{\partial r_{1}}{\partial x}=0, \quad \frac{\partial r_{2}}{\partial t}+r_{1} \frac{\partial r_{2}}{\partial x}=0,
$$

где

$$
r_{1}=-A_{13}-\sqrt{A_{13}^{2}+A_{11}}, \quad r_{2}=-A_{13}+\sqrt{A_{13}^{2}+A_{11}} .
$$

В частности, для уравнения Борна-Инфельда инварианты Римана имеют вид [1], [4]:

$$
r_{1}=-\left(\frac{p \lambda+\sqrt{1+p^{2}-\lambda^{2}}}{1+p^{2}}\right), \quad r_{2}=\frac{-p \lambda+\sqrt{1+p^{2}-\lambda^{2}}}{1+p^{2}} .
$$

ЗАмЕчАниЕ 1 . В дальнейшем для сокращения записи будем называть класс $A$-гиперболических и класс $A$-эллиптических уравнений сокращенно $A$-уравнениями.

1. Формулировка основного результата. Сформулируем теперь основной результат данной работы.

Теорема 1. Все А-уравнения (3), коэффициенты которых удовлетворяют условиям (5), (7) и дополнительному условию

$$
\frac{\partial^{2} A_{13}}{\partial \lambda^{2}}=0
$$

допускают следующий закон сохранения второго порядка:

$$
\frac{\partial}{\partial t}\left[S(p, \lambda)\left(\left(U_{x t}\right)^{2}+A_{11} \cdot\left(U_{x x}\right)^{2}\right)\right]-\frac{\partial}{\partial x}\left[2 S(p, \lambda)\left(A_{11} \cdot U_{x x} \cdot U_{x t}+A_{13} \cdot\left(U_{x t}\right)^{2}\right)\right]=0,
$$


где функиия $S(p, \lambda)$ определяется формулой

$$
S(p, \lambda)=\frac{1}{A_{11}} \exp \left[-4 \int K_{1}(p) d p\right]
$$

а функция $K_{1}(p, \lambda)$ связана с представлением коэфбичиента $A_{13}$ :

$$
A_{13}(p, \lambda)=K_{1}(p) \lambda+K_{2}(p) .
$$

2. Доказательство основного результата. Предварительно выведем формулы, определяющие коэффициенты $A_{13}(p, \lambda)$ для всех $A$-уравнений при дополнительном условии (10).

Для этого записываем условие совместности для системы уравнений (7) с учетом исследуемого класса уравнений (3). После вычислений при учете условия (10) получим следующее уравнение относительно коэффициента $A_{13}$ :

$$
\frac{\partial^{2} A_{13}}{\partial p^{2}}+2 A_{13} \frac{\partial^{2} A_{13}}{\partial p \partial \lambda}+4 \frac{\partial^{2} A_{13}}{\partial \lambda} \cdot \frac{\partial^{2} A_{13}}{\partial p}+4 A_{13}\left(\frac{\partial A_{13}}{\partial \lambda}\right)^{2}=0 .
$$

Подставим теперь в (14) формулу (13): после "расщепления" полученного уравнения по переменной $\lambda$ получим следующую систему двух обыкновенных дифференциальных уравнений относительно $K_{1}(p), K_{2}(p)$ :

$$
\begin{gathered}
K_{1}^{\prime \prime}+6 K_{1} \cdot K_{1}^{\prime}+4 K_{1}^{3}=0, \\
K_{2}^{\prime \prime}+4 K_{1} \cdot K_{2}^{\prime}+\left(4 K_{1}^{2}+2 K_{1}^{\prime}\right) K_{2}=0, \\
K_{i}^{\prime}=\frac{d K_{i}}{d p}, \quad i=1,2 .
\end{gathered}
$$

Уравнение (15) является частным случаем известного уравнения Льенарда [8; с. 232]

$$
y^{\prime \prime}+f(y) y^{\prime}+g(y)=0,
$$

где $f(y), g(y)$ - функции из достаточно широкого класса непрерывных функций, и может быть проинтегрировано приведением к уравнению Абеля.

Кроме того, уравнение (15) может быть с помощью специальных подстановок преобразовано к линейному уравнению второго порядка [9; с. 278]. Полное интегрирование системы (15), (16) приведено в работе [10; с. 59] в связи с решением несколько другой задачи. Приведем здесь только необходимое для дальнейшего изложения решение уравнения (15). Это уравнение имеет общее решение

$$
K_{1}(p)=\frac{p+C_{1}}{\left(p+C_{1}\right)^{2}+C_{2}}
$$

и решение, которое не получается из общего решения

$$
K_{1}(p)=\frac{1}{2\left(p+C_{1}\right)},
$$

$C_{1}, C_{2}$ - две произвольные постоянные. 
Теперь переходим к выводу формулы (12). Выполним дифференцирование в законе сохранения (11). После вычислений с учетом уравнения (3) получим следующее выражение:

$$
l_{1} \cdot U_{x x x}+l_{2} \cdot U_{x x t}+l_{3}\left(U_{x x}\right)^{3}+l_{4}\left(U_{x t}\right)^{3}+l_{5}\left(U_{x x}\right)^{2} \cdot U_{x t}+l_{6}\left(U_{x t}\right)^{2} \cdot U_{x x}=0 .
$$

Здесь $l_{i}=l_{i}\left(U_{x t}, U_{x x}, p, \lambda\right), i=1,2$, тождественно обращаются в нуль. Остальные коэффициенты будут следующими:

$$
\begin{aligned}
& l_{3}=\left(A_{11}\right)^{2} \frac{\partial S}{\partial \lambda}+A_{11}\left(\frac{\partial A_{11}}{\partial \lambda}\right) S \\
& l_{4}=\frac{\partial S}{\partial p}+2 \frac{\partial A_{13}}{\partial \lambda} S \\
& l_{5}=2 A_{11} \cdot A_{13} \frac{\partial S}{\partial \lambda}-A_{11} \frac{\partial S}{\partial p}+S\left(\frac{\partial A_{11}}{\partial p}+2 A_{13} \frac{\partial A_{11}}{\partial \lambda}\right), \\
& l_{6}=-A_{11} \frac{\partial S}{\partial \lambda}-2 A_{13} \frac{\partial S}{\partial p}+2 S \frac{\partial A_{13}}{\partial p} .
\end{aligned}
$$

Поскольку $l_{k}, k=3, \ldots, 6$, зависят только от $p, \lambda$, для удовлетворения уравнения (18) необходимо положить $l_{k}=0, k=3, \ldots, 6$. Тогда из первых двух уравнений (19) получаем систему уравнений

$$
\begin{aligned}
& \frac{\partial S}{\partial p}=-2 \frac{\partial A_{13}}{\partial \lambda} S \\
& \frac{\partial S}{\partial \lambda}=-\frac{1}{A_{11}} \cdot \frac{\partial A_{11}}{\partial \lambda} S, \quad A_{11} \neq 0 .
\end{aligned}
$$

Остальные уравнения $l_{j}=0, j=3,4$, являются следствием системы (20) при учете $(7)$.

Интегрируя систему (20) с учетом (13), получим формулу (12).

\section{3. Примеры.}

ПримеР 1. Уравнение Борна-Инфельда (2). Для этого уравнения будем иметь

$$
A_{11}=\frac{1-\lambda^{2}}{1+p^{2}}, \quad A_{13}=\frac{p}{1+p^{2}} \lambda .
$$

С учетом (13) получим

$$
K_{1}(p)=\frac{p}{1+p^{2}}, \quad K_{2}(p)=0
$$

Интегрируя систему (20), получим

$$
S=\frac{1}{\left(1-\lambda^{2}\right)\left(1+p^{2}\right)}, \quad \lambda \neq 1 .
$$

Закон сохранения будет следующий:

$$
\frac{\partial E_{1}}{\partial t}+\frac{\partial G_{1}}{\partial x}=0
$$


878

$$
\begin{aligned}
\frac{\partial}{\partial t}[ & \left.\frac{\left(U_{x t}\right)^{2}}{\left(1-U_{t}^{2}\right)\left(1+U_{x}^{2}\right)}+\frac{\left(U_{x x}\right)^{2}}{\left(1+U_{x}^{2}\right)}\right] \\
& \quad-\frac{\partial}{\partial x}\left[\frac{2}{1+U_{x}^{2}} \cdot U_{x x} \cdot U_{x t}+\frac{2 U_{x} \cdot U_{t}}{\left(1-U_{t}^{2}\right)\left(1+U_{x}^{2}\right)}\left(U_{x t}\right)^{2}\right]=0 .
\end{aligned}
$$

Если на решениях уравнения Борна-Инфельда (2) выполняется условие

$$
\left|U_{t}\right|<1
$$

то функция $E_{1}$ в $(22)$ будет положительной (плотность закона сохранения).

ПримеР 2. Рассмотрим уравнение минимальных и максимальных поверхностей в пространстве Минковского [11; с. 5-21]

$$
\left(\left(U_{x}\right)^{2}-1\right) U_{t t}-2 U_{x} \cdot U_{t} \cdot U_{x t}+\left(\left(U_{t}\right)^{2}-1\right) U_{x x}=0
$$

Это уравнение в соответствии с (6) и (8) будет принадлежать к гиперболическому типу в области

$$
\left(U_{t}\right)^{2}+\left(U_{x}\right)^{2}>1
$$

а в области

$$
\left(U_{t}\right)^{2}+\left(U_{x}\right)^{2}<1
$$

будет принадлежать к эллиптическому типу. Предполагая, что на решениях уравнения (23) $\left|U_{x}\right| \neq 1,\left|U_{t}\right| \neq 1$, можно получить

$$
K_{1}(p)=\frac{p}{p^{2}-1}, \quad K_{2}(p)=0, \quad S(p, \lambda)=\frac{1}{\left(1-p^{2}\right)\left(\lambda^{2}-1\right)} .
$$

Закон сохранения (11) для уравнения (24) будет таким:

$$
\begin{aligned}
& \frac{\partial}{\partial t}\left[\frac{1}{\left(1-\left(U_{x}\right)^{2}\right)\left(\left(U_{t}\right)^{2}-1\right)}\left(U_{x t}\right)^{2}+\frac{1}{\left(1-\left(U_{x}\right)^{2}\right)^{2}}\left(U_{x x}\right)^{2}\right] \\
& \quad+\frac{\partial}{\partial x}\left[\frac{2 U_{x} \cdot U_{t}}{\left(1-\left(U_{x}\right)^{2}\right)\left(\left(U_{t}\right)^{2}-1\right)}\left(U_{x t}\right)^{2}-\frac{2}{\left(1-\left(U_{x}\right)^{2}\right)^{2}} U_{x x} \cdot U_{x t}\right]=0 .
\end{aligned}
$$

ПримеР 3. Рассмотрим уравнение, описывающее потенциал скоростей в случае одномерного нестационарного движения газа Чаплыгина [4]

$$
U_{t t}+2 U_{x} \cdot U_{x t}-\left(1+2 U_{t}\right) U_{x x}=0 .
$$

В этом примере $A_{13}=-p$, поэтому

$$
K_{1}(p)=0, \quad K_{2}(p)=-p, \quad S=\frac{\lambda}{1+2 \lambda} .
$$

Закон сохранения (11) здесь будет таким:

$$
\frac{\partial}{\partial t}\left[\frac{\left(U_{x t}\right)^{2}}{1+2 U_{t}}+\left(U_{x x}\right)^{2}\right]+\frac{\partial}{\partial x}\left[\frac{2 U_{x}}{1+2 U_{t}}\left(U_{x t}\right)^{2}-2 U_{x x} \cdot U_{x t}\right]=0 .
$$


Пример 4. В качестве примера $A$-эллиптического уравнения найдем закон сохранения (11) для классического уравнения минимальных поверхностей [12], которое запишем в стандартной форме в переменных $x, y$ :

$$
\left(1+U_{y}^{2}\right) U_{x x}-2 U_{x} \cdot U_{y} \cdot U_{x y}+\left(1+U_{x}^{2}\right) U_{y y}=0 .
$$

Чтобы воспользоваться формулой (11), положим

$$
t=x, \quad x=y, \quad U_{y}=0 .
$$

Тогда

$$
K_{1}=\frac{U_{y}}{1+U_{y}^{2}}=\frac{p}{1+p^{2}}, \quad K_{2}=0
$$

Получим

$$
S=\frac{1}{\left(1+U_{x}^{2}\right)\left(1+U_{y}^{2}\right)} .
$$

Закон сохранения (11) для уравнения (30) будет следующим:

$$
\begin{aligned}
& \frac{\partial}{\partial y}\left[\frac{1}{\left(1+U_{x}^{2}\right)\left(1+U_{y}^{2}\right)}\left(U_{x y}\right)^{2}-\frac{\left(U_{x x}\right)^{2}}{\left(1+U_{x}^{2}\right)^{2}}\right] \\
& \quad+\frac{\partial}{\partial x}\left[\frac{2 U_{x x} \cdot U_{x y}}{\left(1+U_{x}^{2}\right)^{2}}-\frac{2 U_{x} \cdot U_{y}}{\left(1+U_{x}^{2}\right)\left(1+U_{y}^{2}\right)}\left(U_{x y}\right)^{2}\right]=0 .
\end{aligned}
$$

ЗАмЕЧАниЕ 2. Уравнения (2), (27) при условиях

$$
\left|U_{x}\right| \square 1, \quad\left|U_{t}\right| \square 1
$$

совпадают с одномерным волновым уравнением

$$
U_{t t}-U_{x x}=0
$$

Соответственно, законы сохранения (22) и (29) при этих условиях будут совпадать с законом сохранения второго порядка для волнового уравнения (33)

$$
\frac{\partial}{\partial t}\left[\left(U_{x t}\right)^{2}+\left(U_{x x}\right)^{2}\right]-\frac{\partial}{\partial x}\left[2 U_{x x} \cdot U_{x t}\right]=0 .
$$

ЗАмЕчаниЕ 3. Уравнения (24), (30) при условиях $\left|U_{x}\right| \square 1,\left|U_{y}\right| \square 1$ (в (24) следует положить $t=x, x=y$ ) будут совпадать с уравнением Лапласа

$$
U_{x x}+U_{y y}=0
$$

Законы сохранения (26) и (32) при этом будут совпадать с законом сохранения второго порядка для уравнения Лапласа (34)

$$
\frac{\partial}{\partial x}\left[\left(U_{y y}\right)^{2}-\left(U_{x y}\right)^{2}\right]-\frac{\partial}{\partial y}\left[2 U_{y y} \cdot U_{x y}\right]=0 .
$$

ЗАмечАниЕ 4. Нетрудно проверить, что во всех примерах функции $K_{i}(p), i=$ 1,2 , определяемые формулами (21), (25), (28), (31) удовлетворяют системе (15), (16). В примере $3 K_{1}(p)=0$ получается из $(17)$ при $C_{2} \rightarrow \infty$. 


\section{4. Заключительные замечания.}

ЗАмЕчАНИЕ 5. Если какое-либо уравнение с частными производными или система таких уравнений допускает законы сохранения, то, как правило, это связано с наличием у данного уравнения (или системы) определенных симметрий: классических, т.е. точечных [13], или обобщенных [2], [3]. В связи с этим обстоятельством полученные в данной статье законы сохранения второго порядка, по-видимому, связаны с некоторыми симметриями системы (9) и соответствующей системы в эллиптической области [10]. Однако работы на эту тему автору не известны.

ЗАмечАниЕ 6 . Закон сохранения (11) связан с нахождением одной функции $S=$ $S\left(U_{x}, U_{t}\right)$. Обобщение этого закона в случае квазилинейных уравнений второго порядка со многими независимыми переменными (например, для многомерного уравнения Борна-Инфельда [14]) связано с преодолением больших вычислительных трудностей, так как возникает задача об исследовании на совместность переопределенной системы уравнений относительно нескольких функций $S_{1}, S_{2}, \ldots, S_{k}$, зависящих от первых производных функции $U$.

ЗАмЕчАНИЕ 7. Отметим, наконец, следующий интересный факт. Условие (23) означает, что на таких решениях уравнение Борна-Инфельда будет $t$-гиперболическим по Петровскому [15], а задача Коши для уравнения Борна-Инфельда, впервые исследованная в работе [16], будет иметь классическое решение в целом в произвольном характеристическом треугольнике [17].

Выражаю сердечную благодарность С. И. Похожаеву, который поставил задачу о нахождении законов сохранения второго порядка для уравнения Борна-Инфельда.

\section{СПИСОК ЦИТИРОВАННОЙ ЛИТЕРАТУРЫ}

[1] О.Ф. Меньших, "О законах сохранения и преобразованиях Беклунда, связанных с уравнением Борна-Инфельда", Матем. заметки, 77:4 (2005), 551-565.

[2] Н. Х. Ибрагимов, Группы преобразований в математической физике, Наука, М., 1983.

[3] П. Олвер, Приложение групп Ли к дифференииальным уравнениям, Мир, М., 1989.

[4] О.Ф. Меньших, "О взаимодействии финитных уединенных волн для уравнений типа Борна-Инфельда", ТМФ, 79:1 (1989), 16-29.

[5] О.Ф. Меньших, "Об одном обобщении формул Вейерштрасса в теории минимальных поверхностей”, Арифметика и геометрия многообразий, Самарский гос. ун-т, Самаpa, 1992, 93-100.

[6] О.Ф. Меньших, "О задаче Коши и краевых задачах для одного класса систем квазилинейных гиперболических уравнений”, Докл. АН СССР, 314:1 (1990), 105-110.

[7] Б. Л. Рождественский, Н. Н. Яненко, Системы квазилинейных уравнений и их приложения к газовой динамике, Наука, М., 1978.

[8] В. Ф. Зайцев, А. Д. Полянин, Справочник по обыкновенным дифференциалъным уравнениям, Физматлит, М., 2001.

[9] Л.М.Беркович, Факторизачия и преобразования дифференциальных уравнений. Методы и приложения, РХД, М., 2002.

[10] О.Ф. Меньших, Об одном обобщении уравнения Борна-Инфельда, деп. в ВИНИТИ $7920-B 86$.

[11] В. М. Миклюков, "Геометрический анализ поверхностей нулевой средней кривизны", Геометрический анализ и его приложения, Волгоградск. гос. ун-т, Волгоград, 1999, $5-21$. 
[12] Дао Чонг Тхи, А. Т. Фоменко, Минимальные поверхности и проблема Плато, Наука, M., 1987.

[13] Л.В. Овсянников, Групповой анализ дифференциалъных уравнений, Наука, М., 1978.

[14] Б. М. Барбашов, Н. А. Черников, "Решение задачи о рассеянии двух плоских волн в нелинейной скалярной теории поля типа Борна-Инфельда", ЖЖЭТФ, 51:8 (1966), $658-668$.

[15] О.Ф. Меньших, О классификации квазилинейных уравнений второго порядка, деп. в ВИНИТИ 1081-В2005.

[16] Б. М. Барбашов, Н. А. Черников, "Решение и квантование нелинейной двумерной модели типа Борна-Инфельда", ЖЭТФ, 50:5 (1966), 1296-1308.

[17] О.Ф. Меньших, "О взаимодействии уединенных волн для системы уравнений Борна-Инфельда", ТМФ, 84:2 (1990), 181-194.

О. Ф. Меньших

Самарский государственный аэрокосмический университет

им. С. П. Королева (СГАУ)

E-mail: oleg_menshin@mail.ru
Поступило

06.12 .2005 